\title{
SUR LA COIMPOSITION ET LE RENDEMENT ÉNERGÉTIQUE DU LAIT DE FEMIME EN PÉRIODE DE RESTRICTIONS (novembre-décembre 1942) (1)
}

\author{
par \\ Jacques lavagne et Solange Mathieu
}

Nous avons voulu déterminer si le régime alimentaire actuel avait une influence sur la composition et sur la valeur énergétique du lait maternel. Pour cela, nous en avons prélevé chez 13 femmes pendant leur séjour à la maternité, les $5^{\mathrm{e}}, 8^{\mathrm{e}}$ et $11^{\mathrm{e}}$ jours après l'accouchement et obtenu ainsi 34 échantillons au lieu de 39 ; la sécrétion lactée s'est, en effet, dans les deux cas, précocement tarie.

Les échantillons analysés résultent du mélange de prélèvements faits le matin, à midi et le soir. Les dosages effectués ont été les suivants :

\section{Constituants énergétiques.}

1. Matière grasse par la méthode d'Adam [1].

2. Lactose par la méthode de BAUDoIn et LEwin avec défécation au ferrocyanure de zinc [1].

3. La caséine et matières albuminoïdes par la méthode de Mercier [2].

4. L'extrait sec (après un séjour de 4 heures à $+105^{\circ}$ ).

\section{$2{ }^{\circ}$ Constituants minéraux.}

5. Phosphore par la méthode de MacheBce Uf [3].

6. Calcium par la méthode de Velluz et Deschazeaux [8].

Les moyens dont nous disposons ne nous ont pas permis de mesurer la valeur vitaminique de nos laits.

\section{Constituants énergétiques}

1. Des dosages que nous avons pratiqués, on dégage malaisément une règle générale concernant l'évolution des substances énergétiques dans les jours qui suivent l'accouchement; en effet, nous avons $\mathrm{vu}$ :

La matière grasse augmenter, puis diminuer dans 6 cas ;

Diminuer régulièrement dans 3 cas ;

Diminuer, puis augmenter dans 2 cas.

(1) Bulletin de la Société de Chimie Biologique, 1943, XXV, 112. 
Le lactose diminuer, puis augmenter dans 5 cas ;

Augmenter, puis diminuer dans 4 cas;

Diminuer régulièrement dans 2 cas.

La caséine et les matières protéiques :

Diminuer régulièrement dans 6 cas ;

Diminuer, puis augmenter dans 4 cas;

Augmenter, puis diminuer dans 1 cas.

L'extrait sec augmenter, puis diminuer danş 5 cas ;

Diminuer, puis augmenter dans 2 cas;

Augmenter régulièrement dans 2 cas.

Si l'on compare nos résultats avec les normales, nous trouvons par rapport aux chiffres donnés par MARFAN [4] :

La matière grasse 14 fois inférieure, oscillant entre $10 \mathrm{gr}$. 05 et 27 gr. 20 p. 1.000.

5 fois égale ;

15 fois supérieure oscillant entre 32 gr. et 60 gr. 65 . p. 1.000 .

Le lactose 33 fois inférieur, oscillant entre $19 \mathrm{gr} .15$ et $58 \mathrm{gr} .60$ p. 1.000 ;

1 fois supérieur à 66 gr. 95 p. 1.000 .

La caséine et les matières protéiques, 34 fois supérieures, oscillant entre $17 \mathrm{gr}$. 50 et $43 \mathrm{gr} .70 \mathrm{p}$. 1.000 .

L'extrait sec, 6 fois inférieur, oscillant de 104 à 122 grammes p. 1,000 ;

3 fois égal ;

23 fois supérieur, oscillan ${ }^{+}$entre 126 et 152 grammes p. 1.000 ;

2 fois indéterminé.

Il se dégage nettement ici un déficit constant en lactose et une plus-value à peine moins constante en matières protéiques ; ce dernier point ne doit pas surprendre, car nos laits sont encore très voisins du colostrum riche en matières azotées.

On peut évidemment se faire une idée de la valeur d'un lait en comparant aux normales, les chiffres de la matière grasse du lactose, de la caséine et de l'extrait see, mais le calcul du rendement énergétique donne des résultats infiniment plus éloquents. Nous avons repris les chiffres de HEUBNER [10] qui admet, pour 1 litre de lait de femme normal, une valeur énergétique moyenne de 650 à 700 calories avec les coefficients suivants : 9,25 pour la matière grasse; 3,96 pour le lactose ; 5,83 pour les matières azotées (1).

Sur 34 échantillons de lait provenant de 13 jeunes mères, nous avons trouvé le rendement énergétique :

(1) Cette valeur énergétique moyenne a été établie avant la découverte du gynolactose et de l'allolactose par Polonovski et Boulanger. Nos chiffres ayant été calculés sur les mêmes bases que ceux de Heubner conservent done leur valeur. 
25 fois inférieur à la normale, oscillant de 436 à 641 calories, soit un déficit de 2 à 33 p. 100 ;

4 fois égal entre 650 et 700 calories ;

5 fois supérieur, oscillant de 753 à 780 calories.

Une seule de nos femmes a donné un lait constamment supérieur à la normale au point de vue énergétique.

12 échantillons, soit un tiers environ, ont un rendement compris entre 500 et 550 calories, soit un déficit moyen de 21 p. 100 ;

5 échantillons donnent un chiffre inférieur à 500 calories avec un déficit de 33 p. 100 ;

5 échantillons sont compris entre 550 et 600 calories avec un déficit moyen de 12 p. 100 ;

2 échantillons sont compris entre 600 et 650 calories avec un déficit moyen de 3 p. 100.

\section{Il. Rapport phosphocalcique}

1. En ce qui concerne l'évolution du rapport $\mathrm{Ca} / \mathrm{P}$ dans les premiers jours de la lactation, nous avons constaté en général une diminution de la valeur du $5^{\mathrm{e}}$ au $12^{\mathrm{e}}$ jour (10 cas). Dans 2 cas seulement cette évolution s'est faite dans le sens de l'augmentation.

2. En traduisant en $\mathrm{Ca}$ et en $\mathrm{P}$ les valeurs données en $\mathrm{CaO}$ et $\mathrm{P}_{2} \mathrm{O}_{5}$ par Polonovski [5] on trouve comme normales, exprimées en milligrammes par litre $: P=160$ et $\mathrm{Ca}=280$, soit un rapport $\mathrm{Ca} / \mathrm{P}=1,75$. Pour Mme RANDorN [6] la valeur de ce rapport est. de 1,58 .

La comparaison de nos résultats avec ces chiffres nous amène aux-constatations suivantes.

Sur 33 dosages pratiqués :

Le phosphore s'est révélé 19 fois inférieur à la normale, oscillant entre 0,085 et 0,152 ;

5 fois égal ;

9 fois supérieur, oscillant entre 0,179 et 0,242 .

Le calcium s'est révélé 17 fois supérieur, oscillant entre 0,300 et 0,426 ;

4 fois égal ;

12 fois inférieur, oscillant entre 0,194 et 0,272 .

Le rapport $\mathrm{Ca} / \mathrm{P}$ s'est révélé 23 fois supérieur à la normale, oscillant entre 1,84 et 3,55 ;

4 fois égal ;

6 fois inférieur, oscillant entre 1,16 et 1,48 .

Le taux du phosphore est done en général faible, celui du calcium et la valeur du rapport $\mathrm{Ca} / \mathrm{P}$ élevés. 


\section{Conclusions}

Les conclusions que l'on peut tirer de ces quelques dosages sont loin d'être optimistes.

En effet dans plus du tiers des cas, la matière grasse est diminuée, le lactose l'est constamment; il en résulte un déficit calorifique qui porte sur les deux tiers des échantillons analysés, ce déficit étant en moyenne de 21 p. 100 . Rappelors que Mme Randorn et ses collaborateurs [7, 8] avaient constaté en février-mars 1942 que, dans l'ensemble, la valeur calorifique n'était pas influencée par le régime des mères, cependant déficiènt dans bien des cas.

D'autre part, la valeur du rapport Calcium/Phosphore est, dans presque deux tiers des cas, supérieur à la normale, donc nettement rachitigène.

Remarquons enfin que les dosages de matière grasse, caséine, lactose, extrait sec, seuls pratiqués habituellement, sont insuffisants pour se faire une idée précise de la valeur d'un lait de femme. Il est nécessaire, en outre, de calculer sa valeur énergétique au moyen des coefficients indiqués ci-dessus et de déterminer le rapport $\mathrm{Ca} / \mathrm{P}$.

\section{(Hôpital Civil de Versailles.)}

\section{BIBLTOGRAPHIE}

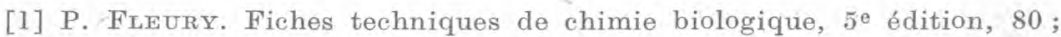
édition Véga.

[2] J. Guiart et L. Grimbert. Diagnostic ehimique, microseopique et parasitologique, 1922, $4^{\mathrm{e}}$ édition, 342, Paris ; Lamare, éditeur.

[3] M.-A. Масневquf, Bull. Soc. Chim. biol., 1926, 8, 464.

[4] A.-B. Marfan. Traité de l'allaitement, 1920, 3 e édition, Paris ; Masson, éditeur.

[5] M. Polonovski. Eléments de Biochimie médicale, 1941, 531, Paris ; Masson, éditeur.

[6] L. Randoin. Bull. Soc. Chim. biol., 1941, 23, 437.

[7] L. Randoin, A. Rossier, J. Boisselot et P. Fournier. C. R. Ac. Méd., 3 nov. 1942.

[8] L. Randoin et A. Raffy. C. R. Ac. Méd., 5 janvier 1943.

[9] L. Venutz et R. Deschaźaux. C. R. Soc. biol., 1930, 104, 977.

[10] Encyclopédie médico-chirurgicale. Pédiatrie, t. I, fasc. 4.026. 